\title{
Transcriptomic Changes During Stage Progression of Mycosis Fungoides
}

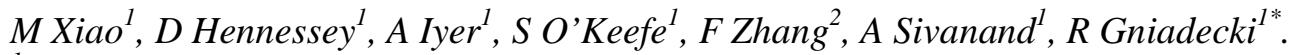 \\ ${ }^{l}$ Division of Dermatology, University of Alberta, Edmonton, AB, Canada \\ ${ }^{2}$ Faculty of Medicine, University of Ottawa, ON, Canada \\ *Address for correspondence: Dr. Robert Gniadecki, 8-112 Clinical Science Building, 11350-83 Ave, \\ Edmonton, AB, T6G 2G3, Canada Tel: (780) 407-1555, Fax: (780) 407-2996, email: \\ r.gniadecki@ualberta.ca
}

Keywords: cutaneous T-cell lymphoma (CTCL), mycosis fungoides (MF), transcriptome analysis, expression profiling

Word count: 3555 (excludes abstracts)

Table count: 0

Figure count: 5

Short title (64/70 characters): Gene Expression Patterns in Early and Advanced Mycosis Fungoides

Conflict of Interest: RG received advisory board honoraria from Kyowa Kirin, Sanofi, Recordati Rare Diseases, and Mallinckrodt and obtained research funding from Sanofi and Sun Pharma.

Funding: This study was supported by grants from the following sources: Canadian Dermatology Foundation (CDF RES0035718), University Hospital Foundation (University of Alberta), Bispebjerg Hospital (Copenhagen, Denmark), Danish Cancer Society (Kræftens Bekæmpelse R124-A7592 Rp12350), and an unrestricted research grant to R Gniadecki from the Department of Medicine, University of Alberta. M Xiao was supported by an Alberta Innovates Summer Research Studentship. A Sivanand was supported by scholarships from the Canadian Institutes of Health Research (CIHR), Alberta Innovates, and the University of Alberta.

These funding organizations had no role in the design or conduct of the research.

\section{What is known:}

- Mycosis fungoides (MF) is the most common cutaneous T-cell lymphoma characterized by a favourable prognosis in the early patch/plaque stage.

- Development of tumors heralds progression to the advanced stage and a significant increase in

What's new: mortality.

- Tumor progression is associated with recurrent mutations which can be linked to the upregulation of signaling pathways controlling cell proliferation, survival, mitosis, and DNA repair.

- Percolation of malignant cells between lesions and tumor self-seeding is likely to mediate stage progression in MF.

\section{Translational message:}

The development of cutaneous tumors in MF heralds stage progression and increased mortality. MF tumors show upregulation in several targetable signaling pathways involved in cell proliferation and survival. Ectopic expression of cancer-testis genes may explain mitotic aberrations in MF tumors. We also propose that high-grade malignant cells can spread hematogenously and seed other skin lesions (tumor self-seeding). Early, aggressive treatment of tumors may prevent tumor self-seeding and improve patient prognosis. 


\section{Abstract}

2 Background: Mycosis fungoides (MF) is the most common cutaneous T cell lymphoma, which in the

3 early patch/plaque stages runs an indolent course. However, $25 \%$ of MF patients develop skin tumors, a

4 hallmark of progression to the advanced stage and associated with high mortality. The mechanisms

5 involved in stage progression are poorly elucidated.

6 Methods: We performed whole-transcriptome and whole-exome sequencing of malignant MF cells from

7 skin biopsies obtained by laser-capture microdissection. We compared three types of MF lesions: early-

8 stage plaques (ESP, $n=12$ ), and plaques and tumors from patients in late-stage disease (late-stage plaques,

9 LSP, n=10, and tumors, TMR, n=15). Gene Ontology (GO) and KEGG analysis were used to determine

10 pathway changes specific for different lesions which we linked to the recurrent somatic mutations

11 overrepresented in MF tumors.

12 Results: The key upregulated pathways during stage progression were those related to cell proliferation 13 and survival (MEK/ERK, Akt-mTOR), Th2/Th9 signaling (IL4, STAT3, STAT5, STAT6), meiomitosis

14 (CT45A1, CT45A3, STAG3, GTSF1, and REC8) and DNA repair (PARP1, MYCN, OGG1). Principal

15 coordinate clustering of the transcriptome revealed extensive gene expression differences between early

16 (ESP) and advanced-stage lesions (LSP and TMR). LSP and TMR showed remarkable similarities at the

17 level of the transcriptome, which we interpreted as evidence of cell percolation between lesions via

18 hematogenous self-seeding.

19 Conclusion: Stage progression in MF is associated with Th2/Th9 polarization of malignant cells,

20 activation of proliferation, survival, as well as increased genomic instability. Global transcriptomic

21 changes in multiple lesions are probably caused by hematogenous cell percolation between discrete skin

22 lesions. 


\section{Introduction}

Mycosis fungoides (MF) is the most prevalent form of primary cutaneous T-cell lymphoma

3 (CTCL), a heterogeneous group of extranodal lymphoproliferative disorders that involve the skin. ${ }^{1}$ Early4 stage (IA-IIA) disease is characterized by erythematous papules, patches, and plaques, and the majority of 5 patients (80-90\%) experience an indolent disease course with slow progression over years or even 6 decades. ${ }^{2-5}$ However, $~ 25 \%$ of MF patients progress to advanced-stage (IIB-IV) disease, distinguished by 7 the appearance of skin tumors, extracutaneous dissemination to lymph nodes, blood, and viscera, as well 8 as a dramatic reduction in survival (decrease in 5-year survival from approximately $80 \%$ to $26 \%$ ). ${ }^{2-5}$ The 9 molecular mechanisms underpinning stage progression in MF remain largely unknown. Moreover, it has 10 not been explained how the emergence of even a single tumor in a relatively small area of the skin 11 impacts the global evolution and prognosis of MF. It would be logical to propose that the more aggressive 12 cancer cells in skin tumors spread hematogenously to other skin areas, increasing tumor load and the 13 clinical impact of the malignancy. However, the putative hematogenous percolation of tumor cells 14 between different lesions has not been investigated because MF cells have been considered to represent mutated tissue-resident T-cells which tend to remain arrested in the defined areas of the skin and do not recirculate via lymphatics and the lymph nodes like the transient passenger T-cells. ${ }^{6} \mathrm{We}$ reasoned that the

17 hypothesis that MF cells traffic between skin lesions can be addressed by comparing transcriptomic profiles of skin lesions in different clinical stages of MF.

Previous analysis of the mutational landscape of CTCL revealed the emergence of numerous subclones and very complex patterns of clonal driver mutations, with little consistency among patients. ${ }^{7-11}$ Over the past decade, studies have focused on the analysis of the transcriptome in an attempt to

22 characterize cancer driver genes and signaling pathways, but the results have been discordant. ${ }^{12}$ This is in part due to the high degree of intratumoral heterogeneity as well as the lack of standard approaches in both sample selection and experimental design that hinders direct comparison between different transcriptome studies. ${ }^{12}$ Many studies pooled samples from MF and the leukemic CTCL (Sézary syndrome, SS) in spite of the data indicating that these entities may represent separate diseases ${ }^{6,13}$ and few transcriptome studies account for the stage of disease and the morphology of skin lesions (e.g., patch, plaque, or tumor). Moreover, the samples used for transcriptomics were usually crude skin biopsies, in which the proportion of neoplastic cells is quite low, often below $30 \%{ }^{7}$

In this study, we compared transcriptome profiles of early and advanced MF to reveal potential mechanisms of disease progression. To capture stage-dependent expression changes, we classified MF skin lesions according to the patient's clinical disease stage (early [stage I] vs advanced [stage $\geq$ IIB]) and 
the morphology of the lesion (plaque vs tumor). To circumvent earlier limitations, we used laser capture microdissection of neoplastic cell clusters to eliminate signal from normal cells. We were able to confirm some of the previously reported pathways associated with advanced-stage MF and identified new transcriptomic changes, such as upregulation of pseudo-meiotic processes (meiomitosis) that may be involved in tumor progression. Moreover, we found that stage progression is associated with global changes in transcriptomic profiles affecting multiple MF lesions which we interpret as evidence of cancer cell percolation proposed by us previously ${ }^{14}$ and analogous to tumor self-seeding. ${ }^{15,16}$

\section{Methods}

\section{Samples, whole transcriptome, and whole-exome sequencing}

Ethics approval was obtained under the application HREBA.CC-19-0435 from the Institutional

11 Review Board at the University of Alberta. 37 laser-microdissected skin lesions were collected from 23

12 adult patients with a diagnosis of MF in stages IA to IVB (Supplementary Table S1), as previously

13 described $^{7}$. RNA was isolated from the microdissected tissues using AllPrep DNA/RNA Micro kit from

14 Qiagen (Hilden, Germany). For samples MF4_2, MF4_3, MF5_1, MF7_1, MF7_2, MF11, MF11_1,

15 MF19_1, MF19_2, and MF19_3 rRNA was inhibited using NEBNext rRNA Depletion kit (NEB,

16 Ipswich, Massachusetts, USA) and later processed using NEBNext Ultra II Directional RNA Library Prep

17 kit for Illumina (Ipswich, Massachusetts, USA). The rest of the samples in the study were processed using

18 Ovation Solo RNA-seq system (Nugen, Redwood city, CA, USA). The manufacturer's instructions were

19 followed and no modifications were made to the protocols. The RNA libraries were sequenced on an

20 Illumina HiSeq 1500 sequencer using paired-end 150 kits (cat\# PE-402-4002) (Hiseq PE rapid cluster kit

21 V2) or NovaSeq 6000 S4 reagent kit 300 cycles (cat\# 20012866). Previously published whole-exome

22 sequencing from the same samples was used for the analysis of single nucleotide variants, as described. ${ }^{17}$

Skin biopsies were categorized into three lesion types according to the clinical stage and morphology of the lesion at the time of collection. Plaque lesions sampled from patients in early-stage MF (IA-IIA) were designated as ESP (early-stage plaque, $n=12$ ). Patients with advanced-stage disease

26 ( $\geq$ IIB) have two types of skin lesions, the TMR (tumors, $n=15$ ) and the plaques which either persist from

27 the early stages (late-stage plaques, LSP, $n=10$ ). There was no significant difference in tumor cell fraction

28 between microdissected samples from different lesion types. 
The bioinformatics pipeline involved a series of aligners and statistical methods shown in Figure 1. All programs were used with default or developer-supplied settings unless otherwise specified. Code is available at https://github.com/d-henness/deseq2_project. Paired fastq files were preprocessed using fastp (v 0.19.7) with the --detect_adapter_for_pe flag enabled. ${ }^{18}$ The resulting fastq files were then aligned and quantified using RSEM (v. 1.3.3). ${ }^{19}$ RSEM was used with Bowtie 2 (v. 2.4.1) ${ }^{20}$ as its backend aligner and an index constructed using Ensembl's 98 human genome annotation. ${ }^{21}$ This yielded gene expression levels in units of transcripts per million (TPM).

Unsupervised Principal Coordinates Analysis (PCoA) was performed using edgeR (R package, v.

11 subspace. PCoA provides insights into the association between samples based on gene expression patterns 12 and detects the formation of clusters among individual specimens. The DESeq2 program, implemented as 13 a package in R (v. 1.28.1) ${ }^{23}$ was used to identify differentially expressed genes (DEGs) that differed

14 significantly between pairwise disease stages $(\log 2$ fold change $(\log 2 \mathrm{FC})>|1|)$. Benjamini-Hochberg 15 correction was used to correct for multiple comparisons, with a standard false discovery cut-off (FDR) of $<0.05$.

To gain further insight into the functional processes differing between lesions, we used all of the

18 available gene expression data (cutoff-free) and performed gene set analysis using the Generally

19 Applicable Gene Set Enrichment (GAGE) method implemented as a package in R (v. 2.37.0). ${ }^{24} \mathrm{We}$

20 focused on Gene Ontology (GO) Biological Process (BP) terms ${ }^{25}$ and Kyoto Encyclopedia of Genes and

21 Genomes (KEGG) pathways. ${ }^{26}$ In both databases, Benjamini-Hochberg correction was used to correct for

22 multiple comparisons (FDR <0.05). Visual data representations were created using the R packages

23 "ggplot2" (v. 3.3.1), "pheatmap" (v. 1.0.12), and 'EnhancedVolcano' (v. 1.6.0). ${ }^{27}$

\section{Data availability}

25 The exome sequencing data is available on dbGaP under accession number phs001877.v1.p1. The RNA 26 sequencing fastq files are available on Sequence Read Archive (SRA) under accession number....

\footnotetext{
${ }^{1}$ The accession number will be provided upon acceptance of the paper. For review purposes the RNA sequencing data can be requested via contacting the corresponding author.
} 


\section{Results}

\section{Changes in transcriptome signatures during stage progression of MF}

Principal coordinates analysis (PCoA) was used to examine the associations between our samples (12 ESP, 10 LSP, 15 TMR) and revealed three clusters (Figure 2A). Cluster A contained the majority of ESP samples (11/12, or 91.7\%) whereas the majority of advanced-stage samples (LSP and TMR) were distributed across clusters B1 and B2 (18/25, or 72\% consisting of 8/10 LSP samples and 10/15 TMR samples). This suggests the transcriptome profile of ESP is distinct from the late-stage disease whereas the expression patterns among advanced lesions (LSP and TMR) are similar. Thus, the MF transcriptomic profile reflects the stage of the disease (advanced vs early) rather than the morphology of the lesion (plaque vs tumor) and indicates that significant global alterations in gene expression occur with progression to advanced-stage disease.

Next, we performed pairwise comparative analyses of the expression profiles between ESP, LSP,

13 and TMR samples to identify differentially expressed genes (DEGs) between different lesion types.

14 Differential expression analysis of TMR vs ESP (reference) lesions identified 1,154 DEGs (fold

15 change $>2$; adjusted $\mathrm{P}<0.05$ ), of which 908 DEGs were significantly up-regulated and 246 DEGs were

16 downregulated in TMR compared to ESP (Figure 2B). Fewer DEGs were detected for the comparisons of

17 LSP to ESP (26 DEGs, 18 upregulated in LSP, Figure 2C) and TMR to LSP (29 DEGs, 11 upregulated in

18 TMR, Figure 2D). A list of all differentially regulated genes across all comparisons is presented in Supplementary Tables S2-S4.

To further illustrate the relationship of genes found to be differentially expressed in each pairwise comparison, we used Venn diagram analysis and overlapped the 1,154 DEGs in TMR vs. ESP with the 26 DEGs in LSP vs. ESP (Figure 3). This revealed 1,137 DEGs unique to TMR (895 upregulated, 242 downregulated), 9 DEGs unique to LSP (5 upregulated, 4 downregulated), and a signature of 17 intersecting DEGs common to both LSP and TMR samples (13 upregulated, 4 downregulated), all concordantly altered (i.e., increased in both, or decreased in both). The large overlap in DEGs shared

26 between LSP and TMR (both advanced lesions) compared with ESP corroborates the PCoA results and

27 suggests that progression to advanced-stage affected the global transcriptomic profile of MF skin lesions. 
different MF stages. We used all of the available gene expression data instead of prefiltering for a list of strong DEGs in order to capture coordinated, low-level changes across genes that may belong to common pathways and are regulated by the same transcriptional network.

Our analyses identified 58 enriched GO biological processes and 21 KEGG pathways in TMR compared to ESP (Supplementary Figure S1). The results of GO analyses revealed that TMR lesions were significantly enriched in proliferative signaling ('Ras protein signal transduction', 'activation of MAPK kinase kinase activity', 'interleukin-2 mediated signaling') and immunosuppression processes ('response to interleukin-4' and 'TOR signaling') (Figure 4A). The KEGG pathway analysis results revealed TMR was highly enriched in 'Notch signaling pathway', 'base excision repair', and 'DNA replication'. (Figure 4B). While the heatmaps show a degree of heterogeneity in gene set enrichment scores across the TMR samples, the overall trend in expression of these annotations is upregulated in TMR. The significantly upregulated and downregulated members of each enriched gene set are presented in Supplementary Table S5-S6.

Next, we conducted functional enrichment analyses on LSP vs ESP (reference, Figure 4B). Although only a few individual genes were statistically significant between LSP and ESP, GSA identified 29 GO Biological Processes and 14 KEGG pathways overrepresented in LSP (Supplementary Figure 2). Among the GO and KEGG hits were five overlapping annotations also captured in the comparison between TMR and ESP (Figure 4C). These include three GO terms: 'respiratory burst' (inflammatory response), 'response to interleukin-4' (Th2 response), and 'TOR signaling' (growth and metabolism) as well as two KEGG pathways related to chromatin regulation: 'DNA replication' and 'base excision repair'.

We also identified GO terms and KEGG pathways uniquely enriched in the LSP group compared with ESP (Figure 4C), including three DNA damage removal annotations ('nucleotide-excision repair'

24 [GO], 'mismatch repair' [GO], 'interstrand cross-link repair' [GO]), two T-cell function annotations ('Tcell receptor signaling' [KEGG], 'Activation of JUN kinase activity' [GO]), as well as hits in 'cell cycle' [KEGG] and 'p38MAPK cascade' [GO]. Enrichment of major DNA repair mechanisms in LSP is consistent with the widespread structural genomic alterations including fusion transcripts, insertions, deletions, and copy number variation described in advanced MF. ${ }^{10}$ differences were smaller between LSP and TMR than in any other pairwise comparison, with only four 
1 lesions were significantly upregulated in primary metabolic processes including 'ribosome biogenesis'

2 'biosynthesis of amino acids', and 'hexose metabolic process'.

Other Significant DEGs

Annotation databases like KEGG and GO allow us to collapse complex gene expression data into more manageable biological pathways and functional annotations, but the mapping of genes to pathways is limited by current knowledge of known molecular interaction networks and how gene products interact. To identify potential DEGs that could have been overlooked by functional analysis methods, we manually searched for individual genes that were significantly over-represented in each lesion type to provide a more nuanced understanding of transcriptional networks.

We confirmed overexpression of several genes reported previously in CTCL, such as KIR3DL2 ( $>14$ fold) and KIR3DL4 (>6 fold), the members of the killer cell Ig-like receptor family that inhibit natural killer cell-mediated antitumor cytotoxicity. KIR3DL2 has been implicated in $\mathrm{SS}^{28}$ and transformed $\mathrm{MF}^{29}$ and the anti-KIR3DL2 monoclonal antibody IPH4102 has recently demonstrated 14 promising clinical activity in patients with refractory CTCL. ${ }^{30}$ LSP and TMR had increased levels of $15 G N L Y$ (granulysin, an immune alarmin) and NCRI (natural cytotoxicity triggering receptor 1) whose 16 expression was previously found to increase with the progression of $\mathrm{MF}^{31}$ and in SS patients with high 17 tumor burden ${ }^{32}$.

Among the 29 DEGs between TMR and LSP, we found a $>6$ fold decrease in fractalkine Receptor (CX3CR1). CX3CL1 was shown to control effector T cell retention in inflamed skin and in the lungs in atopic dermatitis and asthma ${ }^{33,34}$ and the decrease in its expression may facilitate escapement of malignant cells from the skin to the circulation in late stages MF.

We found ectopic expression of several meiosis-related cancer-testis antigens (meiCT) in TMR lesions. MeiCT antigens are normally present only in germ cells during oocyte development and spermatogenesis and become transcriptionally silent in normal somatic tissues. Ectopic activation of meiCT is a frequent phenomenon in cancer because global deregulation of epigenetic signaling causes unprogrammed gene activation. ${ }^{35}$ While our results confirm upregulated expression of two previously described meiCT antigens (GTSF1 [gametocyte specific factor 1] ${ }^{36,37}$ and STAG3 [Stromal Antigen 3]) ${ }^{38}$ we also identified increased expression of seven putative CT antigens previously unreported in CTCL, including CT45A1 (Cancer/Testis Antigen Family 45 Member A1), its paralogue CT45A3 (Cancer/Testis

30 Antigen Family 45 Member A3), BRDT (Cancer/Testis Antigen 9), PRAME (Cancer/Testis Antigen

31 130), PAGE5 (Cancer/Testis Antigen Family 16 Member 1), SPAG4 (Sperm Associated Antigen 4), and 
PNMA5 (Paraneoplastic antigen-like 5). These results are in line with the notion that re-expression of a variety of $\mathrm{CT}$ antigens in malignant lymphocytes may contribute to increased genomic instability.

\section{Linking gene mutations to transcriptomic changes}

We examined whether the gene mutation pattern in MF could explain some of the observed alterations in the transcriptome. From the previously published catalogue of mutated genes obtained by whole-exome sequencing ${ }^{17}$ we selected the genes which are frequently mutated in TMR and LSP and more expressed in those advanced-stage lesions compared to ESP. We assumed that those genes would be particularly relevant because mutations (especially damaging mutations) cause a compensatory overexpression of the gene. ${ }^{39} \mathrm{We}$ found that TMR/LSP lesions showed overexpression of mutated genes representing transcription factors and regulators (MED12, MYCN, MYC, EGR3, CIC), genes involved in genome integrity (ERCC2, POLE), the elements of the membrane receptor signalling network of integrated JAK-STAT, MEK/ERK, and PI3K/AKT/mTOR pathway (PLCG1, INPPL1, VAV1, AKT1, PPP2R1A, NFKB2, CARD11, TRAF2, JAK3, STAT3). Mutations in those genes have been linked to the signaling pathways in different cancers as summarized in Figure 5A.

\section{Discussion}

Our study addresses the poorly elucidated question of the mechanisms responsible for the development of tumors in MF, an event that defines the progression from the early to the advanced stage of the disease and which is associated with a dramatic increase in mortality. Among the studies where transcriptomic changes during stage progression were captured ${ }^{37,40-42}$ only two reports ${ }^{36,43}$ and a metaanalysis ${ }^{41}$ compared DEGs directly between early and advanced lesions. The novel aspects of this study is that we not only compared skin lesions in early and late-stage patients, but we included different morphological types of MF lesions (the plaques and the tumors: ESP, LSP, TMR) and used cancer cell enriched, microdissected tissue of the high tumor cell fraction (mean purity [SD] 74.6 [20.4]\%) which allowed us to largely limit the contribution of transcripts originating from non-neoplastic cells in the skin (epidermis, inflammatory cells, fibroblasts, and other stromal cells).

Compared with early-stage plaques (ESP), the tumor (TMR) samples showed an increased genome integrity, JAK-STAT, MAPK/ERK, and PI3K/AKT/mTOR pathways (Figure 5A). Although detailed mechanistic studies are needed to determine the relative importance of those mutations, overlaying the mutated genes on the known signaling pathway elements reveals their likely relevance in cancer progression. It was shown previously that mutated PLCGl increases the survival signaling via NF- 
kB, NFAT and AP-1. ${ }^{11,44}$ Mutated STAT3 was also shown to have a pathogenic role in CTCL. ${ }^{45,46}$.

2 Mutations in $C A R D 11^{9,11}$ and $J A K 3^{2,47}$ are also supposed to increase the growth and survival of malignant

3 T-cells in CTCL and are well-known driver mutations in other hematological malignancies.

Components of MEK/ERK pathway are reproducibly overexpressed in TMR and our mutation data revealed two potentially novel and previously unreported mechanisms of its activation (Figure 5A). MED12 is a tumor suppressor gene which in normal cells inhibits the transforming growth factor $\beta$ (TGF $\beta)$, Akt/mTOR and MAPK/ERK signaling. ${ }^{48-50}$ Interestingly, loss of function of mutated MED12 was shown to be the main driver in certain tumors by activating RAS/MAPK/ERK ${ }^{49-51}$. MED12 was also linked to drug resistance, which might be relevant in CTCL which is notoriously resistant to chemotherapy. Mutations in Capicua (CIC) may amplify MAPK/ERK signaling because CIC is a

11 transcriptional repressor counteracting activation of genes downstream of ERK and contributes to the tumorigenesis of different solid tumors. ${ }^{52-54}$

VAV1 is a hematopoietic-specific RHO guanine exchange factor and its loss of function causes

14 T-cell leukemias by increasing the Notch1-dependent ICN1 signaling due to inhibition of ICN1

15 ubiquitinylation and degradation. ${ }^{55}$ Mutations in VAV1 were reported previously ${ }^{56}$ but were linked to PI-

16 3-kinase signaling rather than Notch signaling. However, previous reports from our lab documenting an

17 increase in Notch1 and its signaling in advanced $\mathrm{MF}^{57}$ confirmed by the Notch1 overexpression in TMR seen here, seem to indicate that VAV1-Notch1 axis may play in MF progression.

The notion that MF is a genetically stable tumor and does not hypermutate ${ }^{58}$ has recently been refuted by genome and exome sequencing data showing numerous mutations and subclonal

21 diversification even in early stages. ${ }^{10,17,59}$ The high rate of mutations can be explained in part by our current findings showing dysregulation of major pathways controlling DNA repair mechanisms (nucleotide excision repair, mismatch repair and base excision repair). Although DNA repair mechanisms

24 have not been studied in MF in detail, it is known that mutations affecting DNA repair genes such as

25 POLE, MYCN, or ATM may contribute to genomic instability in CTCL. ${ }^{17,60-62}$

Another, novel mechanism of genomic instability might be related to abnormal regulation of mitosis. We identified ectopic expressions of five testis-specific genes (CT45A1, CT45A3, STAG3,

28 GTSF1, and REC8) which are only expressed in testicular germ cells. Ectopic expression of cancer-testis

29 (CT) antigens was previously found in carcinomas of the bladder, ${ }^{63}$ lung, ${ }^{64} \operatorname{liver}^{65}$ and suspected to play

30 an important role in maintaining cell survival through inhibition of apoptosis (i.e. down regulating p53

31 and p21 tumor suppressor genes) and promotion of chromosomal instability (i.e. generation of doublestrand breaks leading to loss of heterozygosity and chromosomal arrangements). CT genes (SYCPl, 
SYCP3, REC8, SPO11, STAG3, GTSF1) have previously been reported in advanced CTCL and proposed to reflect the reactivation of meiotic and gametogenic programs in mitotic cells. ${ }^{37,38}$ Our data documenting a dramatic increase in CT expression (10-30 fold up-regulation in MF tumors) support the putative role of meiomitosis ${ }^{66,67}$ as a mechanism of genomic instability in MF.

MF is a spatially discontinuous neoplasm composed of circumscribed lesions of the skin. This calls for a question of whether those lesions evolve in isolation or whether malignant cells percolate between the lesions. Clinical observations support the latter notion; it is known that the development of the tumor in one area of the skin changes the clinical course to be more unfavourable and that those patients are more likely to develop additional tumors. We have therefore hypothesized that high-grade malignant cells in the tumors escape to the circulation and may metastatically seed the skin - either to the

11 already existing lesions or to the new, previously uninvolved areas. The comparisons of global expression

12 profiles of ESP, LSP and TMR seem to confirm our hypothesis. The principal component clustering of

13 transcriptomes showed that LSP and TMR overlapped in two clusters (clusters B1 and B2, Figure 2) and

14 separated well from ESP. The same was previously seen on the mutational level: LSP resembled tumors

15 and differed from the ESP. ${ }^{17,68}$ This shows that stage progression is associated with global genomic and

16 transcriptomic changes in all skin lesions, in spite of the fact that the tumor formation is only present in a

17 discrete skin area. Thus, cancer cells in MF are likely to percolate through the skin, occasionally seeding

18 new areas and the existing lesions, the latter case resembling tumor self-seeding or cell exchange between

19 distant metastases (Figure 5B). ${ }^{15,16,69,70}$ It is conceivable that early eradication of emerging tumors (e.g. by

20 aggressive radiotherapy) would limit hematogenous percolation of malignant cells and retard the

21 colonization of the skin with high-grade mutated cells. 


\section{Acknowledgements}

We extend our gratitude to Dr. Thomas Salopek, Mrs. Rachel Doucet, and the nursing staff of

3 Kaye Edmonton Clinic for their assistance with sample collection. This study was supported by grants

4 from the following sources: Canadian Dermatology Foundation (CDF RES0035718), University Hospital

5 Foundation (University of Alberta), Bispebjerg Hospital (Copenhagen, Denmark), Danish Cancer Society

6 (Kræftens Bekæmpelse R124-A7592 Rp12350) and an unrestricted research grant to R.G. from the

7 Department of Medicine, University of Alberta. M.X. was supported by an Alberta Innovates Summer

8 Research Studentship.

\section{Author's contributions}

M.X. contributed to study design, conducted bioinformatics, analyzed the data, prepared the

12 figures, and wrote the manuscript. RG designed the experiments, supervised data analysis, prepared the

13 figures, and wrote the manuscript. DH and FZ conducted bioinformatics analysis. AI and SO performed

14 wet-lab experiments. AS contributed to writing and editing the manuscript. All authors reviewed all

15 versions of the paper and approved the final version of this manuscript. 


\section{References}

21 Willemze R. Mycosis fungoides variants-clinicopathologic features, differential diagnosis, and 3 treatment. Semin Cutan Med Surg 2018; 37:11-7.

42 Johnson VE, Vonderheid EC, Hess AD, et al. Genetic markers associated with progression in early $5 \quad$ mycosis fungoides. J Eur Acad Dermatol Venereol 2014; 28:1431-5.

63 Talpur R, Singh L, Daulat S, et al. Long-term outcomes of 1,263 patients with mycosis fungoides $7 \quad$ and Sézary syndrome from 1982 to 2009. Clin Cancer Res 2012; 18:5051-60.

84 Bernengo MG, Quaglino P, Novelli M, et al. Prognostic factors in Sézary syndrome: a multivariate

9 analysis of clinical, haematological and immunological features. Ann Oncol 1998; 9:857-63.

10

5 Diamandidou E, Colome M, Fayad L, et al. Prognostic factor analysis in mycosis fungoides/Sézary syndrome. J Am Acad Dermatol 1999; 40:914-24.

6 Campbell JJ, Clark RA, Watanabe R, Kupper TS. Sezary syndrome and mycosis fungoides arise from distinct T-cell subsets: a biologic rationale for their distinct clinical behaviors. Blood 2010; 116:767-71.

7 Iyer A, Hennessey D, O'Keefe S, et al. Clonotypic heterogeneity in cutaneous T-cell lymphoma (mycosis fungoides) revealed by comprehensive whole-exome sequencing. Blood Adv 2019; 3:1175.

8 Chang L-W, Patrone CC, Yang W, et al. An Integrated Data Resource for Genomic Analysis of Cutaneous T-Cell Lymphoma. J Invest Dermatol 2018; 138:2681-3.

9 da Silva Almeida AC, Abate F, Khiabanian H, et al. The mutational landscape of cutaneous T cell lymphoma and Sézary syndrome. Nat Genet 2015; 47:1465-70.

10 Choi J, Goh G, Walradt T, et al. Genomic landscape of cutaneous T cell lymphoma. Nat Genet 2015; 47. doi:10.1038/ng.3356.

11 Wang L, Ni X, Covington KR, et al. Genomic profiling of Sézary syndrome identifies alterations of key T cell signaling and differentiation genes. Nat Genet 2015; 47:1426-34.

12 Dulmage BO, Geskin LJ. Lessons learned from gene expression profiling of cutaneous T-cell lymphoma. Br J Dermatol 2013; 169:1188-97.

13 van Doorn R, van Kester MS, Dijkman R, et al. Oncogenomic analysis of mycosis fungoides reveals major differences with Sezary syndrome. Blood 2009; 113:127-36.

14 Iyer A, Hennessey D, O'Keefe S, et al. Skin colonization by circulating neoplastic clones in cutaneous T-cell lymphoma. Blood 2019; 134:1517-27.

15 Kim M-Y, Oskarsson T, Acharyya S, et al. Tumor self-seeding by circulating cancer cells. Cell 2009; 139:1315-26.

16 Massagué J, Obenauf AC. Metastatic colonization by circulating tumour cells. Nature 2016; 529:298-306.

17 Iyer A, Hennessey D, O' Keefe S, et al. Branched evolution and genomic intratumor heterogeneity in the pathogenesis of cutaneous T-cell lymphoma. Blood Adv 2020; 4:2489-500. 
118 Chen S, Zhou Y, Chen Y, Gu J. fastp: an ultra-fast all-in-one FASTQ preprocessor. Bioinformatics 2018; 34:1884-90.

19 Li B, Dewey CN. RSEM: accurate transcript quantification from RNA-Seq data with or without a reference genome. BMC Bioinformatics 2011; 12. doi:10.1186/1471-2105-12-323.

20 Langmead B, Salzberg SL. Fast gapped-read alignment with Bowtie 2. Nat Methods 2012; 9:357-9.

21 Yates AD, Achuthan P, Akanni W, et al. Ensembl 2020. Nucleic Acids Res 2019; 48:D682-8.

22 Robinson MD, McCarthy DJ, Smyth GK. edgeR: a Bioconductor package for differential expression analysis of digital gene expression data. Bioinformatics 2010; 26:139-40.

23 Love MI, Huber W, Anders S. Moderated estimation of fold change and dispersion for RNA-seq data with DESeq2. Genome Biol 2014; 15:550.

1124 Luo W, Friedman MS, Shedden K, et al. GAGE: generally applicable gene set enrichment for pathway analysis. BMC Bioinformatics 2009; 10:161.

25 The Gene Ontology Consortium, Ashburner M, Ball CA, et al. Gene Ontology: tool for the unification of biology. Nat Genet 2000; 25:25.

26 Kanehisa M, Goto S. KEGG: kyoto encyclopedia of genes and genomes. Nucleic Acids Res 2000; 28. doi:10.1093/nar/28.1.27.

27 K Blighe, S Rana, M Lewis. EnhancedVolcano: Publication-ready volcano plots with enhanced colouring and labeling. https://github.com/kevinblighe/EnhancedVolcano [accessed on 2 January 2021].

28 Michel L, Jean-Louis F, Begue E, et al. Use of PLS3, Twist, CD158k/KIR3DL2, and NKp46 gene expression combination for reliable Sézary syndrome diagnosis. Blood 2013; 121:1477-8.

29 Ortonne N, Le Gouvello S, Tabak R, et al. CD158k/KIR3DL2 and NKp46 are frequently expressed in transformed mycosis fungoides. Exp Dermatol 2012; 21:461-3.

30 Bagot M, Porcu P, Marie-Cardine A, et al. IPH4102, a first-in-class anti-KIR3DL2 monoclonal antibody, in patients with relapsed or refractory cutaneous T-cell lymphoma: an international, firstin-human, open-label, phase 1 trial. Lancet Oncol 2019; 20:1160-70.

31 Shareef MM, Elgarhy LH, Wasfy RE-S. Expression of Granulysin and FOXP3 in Cutaneous T Cell Lymphoma and Sézary Syndrome. Asian Pac J Cancer Prev 2015; 16:5359-64.

32 Bensussan A, Remtoula N, Sivori S, et al. Expression and function of the natural cytotoxicity receptor NKp46 on circulating malignant CD4+ T lymphocytes of Sézary syndrome patients. $J$ Invest Dermatol 2011; 131:969-76.

33 Staumont-Sallé D, Fleury S, Lazzari A, et al. CX $\square$ CL1 (fractalkine) and its receptor CX $\square \mathrm{CR} 1$ regulate atopic dermatitis by controlling effector T cell retention in inflamed skin. J Exp Med 2014; 211:1185-96. 
35 Rousseaux S, Debernardi A, Jacquiau B, et al. Ectopic activation of germline and placental genes identifies aggressive metastasis-prone lung cancers. Sci Transl Med 2013; 5:186ra66.

36 Litvinov IV, Tetzlaff MT, Thibault P, et al. Gene expression analysis in Cutaneous T-Cell Lymphomas (CTCL) highlights disease heterogeneity and potential diagnostic and prognostic indicators. Oncoimmunology 2017; 6:e1306618.

37 Litvinov IV, Cordeiro B, Huang Y, et al. Ectopic expression of cancer-testis antigens in cutaneous T-cell lymphoma patients. Clin Cancer Res 2014; 20. doi:10.1158/1078-0432.CCR-14-0307.

38 Gantchev J, Martínez Villarreal A, Xie P, et al. The Ectopic Expression of Meiosis Regulatory Genes in Cutaneous T-Cell Lymphomas (CTCL). Front Oncol 2019; 9. doi:10.3389/fonc.2019.00429.

39 El-Brolosy MA, Stainier DYR. Genetic compensation: A phenomenon in search of mechanisms. PLoS Genet 2017; 13:e1006780.

40 Querfeld C, Leung S, Myskowski PL, et al. Primary T Cells from Cutaneous T-cell Lymphoma Skin Explants Display an Exhausted Immune Checkpoint Profile. Cancer Immunol Res 2018; 6:900-9.

41 van Kester MS, Borg MK, Zoutman WH, et al. A meta-analysis of gene expression data identifies a molecular signature characteristic for tumor-stage mycosis fungoides. J Invest Dermatol 2012; 132:2050-9.

42 Bastidas Torres AN, Cats D, Mei H, et al. Genomic analysis reveals recurrent deletion of JAKSTAT signaling inhibitors HNRNPK and SOCS1 in mycosis fungoides. Genes Chromosomes Cancer 2018; 57:653-64.

43 Tracey L, Villuendas R, Dotor AM, et al. Mycosis fungoides shows concurrent deregulation of multiple genes involved in the TNF signaling pathway: an expression profile study. Blood 2003; 102. doi:10.1182/blood-2002-11-3574.

44 Patel VM, Flanagan CE, Martins M, et al. Frequent and Persistent PLCG1 Mutations in Sézary Cells Directly Enhance PLC $\gamma 1$ Activity and Stimulate NFאB, AP-1, and NFAT Signaling. J Invest Dermatol 2020; 140:380-9.e4.

45 Nielsen M, Kaltoft K, Nordahl M, et al. Constitutive activation of a slowly migrating isoform of Stat3 in mycosis fungoides: tyrphostin AG490 inhibits Stat3 activation and growth of mycosis fungoides tumor cell lines. Proc Natl Acad Sci U S A 1997; 94. doi:10.1073/pnas.94.13.6764.

46 Sommer VH, Clemmensen OJ, Nielsen O, et al. In vivo activation of STAT3 in cutaneous T-cell lymphoma. Evidence for an antiapoptotic function of STAT3. Leukemia 2004; 18:1288-95.

47 McGirt LY, Jia P, Baerenwald DA, et al. Whole-genome sequencing reveals oncogenic mutations in mycosis fungoides. Blood 2015; 126:508-19.

48 Guo X, Wang X-F. A mediator lost in the war on cancer. Cell 2012; 151:927-9.

49 Huang S, Hölzel M, Knijnenburg T, et al. MED12 controls the response to multiple cancer drugs through regulation of TGF- $\beta$ receptor signaling. Cell 2012; 151:937-50.

50 Cani AK, Hovelson DH, McDaniel AS, et al. Next-Gen Sequencing Exposes Frequent MED12 Mutations and Actionable Therapeutic Targets in Phyllodes Tumors. Mol Cancer Res 2015; 13:613- 
9.

51 Guinney J, Ferté C, Dry J, et al. Modeling RAS phenotype in colorectal cancer uncovers novel molecular traits of RAS dependency and improves prediction of response to targeted agents in patients. Clin Cancer Res 2014; 20:265-72.

52 Bunda S, Heir P, Metcalf J, et al. CIC protein instability contributes to tumorigenesis in glioblastoma. Nat Commun 2019; 10:661.

53 Weissmann S, Cloos PA, Sidoli S, et al. The Tumor Suppressor CIC Directly Regulates MAPK Pathway Genes via Histone Deacetylation. Cancer Res 2018; 78:4114-25.

54 Eleveld TF, Schild L, Koster J, et al. RAS-MAPK Pathway-Driven Tumor Progression Is Associated with Loss of CIC and Other Genomic Aberrations in Neuroblastoma. Cancer Res 2018; 78:6297307.

55 Robles-Valero J, Lorenzo-Martín LF, Menacho-Márquez M, et al. A Paradoxical Tumor-Suppressor Role for the Rac1 Exchange Factor Vav1 in T Cell Acute Lymphoblastic Leukemia. Cancer Cell 2017; 32:608-23.e9.

56 Park J, Yang J, Wenzel AT, et al. Genomic analysis of 220 CTCLs identifies a novel recurrent gainof-function alteration in RLTPR (p.Q575E). Blood 2017; 130:1430-40.

57 Kamstrup MR, Gjerdrum LMR, Biskup E, et al. Notch1 as a potential therapeutic target in cutaneous T-cell lymphoma. Blood 2010; 116:2504-12.

58 Assaf C, Sanchez JAA, Lukowsky A, et al. Absence of microsatellite instability and lack of evidence for subclone diversification in the pathogenesis and progression of mycosis fungoides. $J$ Invest Dermatol 2007; 127:1752-61.

59 Iyer A, Hennessey D, O'Keefe S, et al. Independent evolution of cutaneous lymphoma subclones in different microenvironments of the skin. Sci Rep 2020; 10:15483.

60 Woollard WJ, Pullabhatla V, Lorenc A, et al. Candidate driver genes involved in genome maintenance and DNA repair in Sézary syndrome. Blood 2016; 127:3387-97.

61 Rickman DS, Schulte JH, Eilers M. The Expanding World of N-MYC-Driven Tumors. Cancer Discov 2018; 8:150-63.

62 Stagni V, Cirotti C, Barilà D. Ataxia-Telangiectasia Mutated Kinase in the Control of Oxidative Stress, Mitochondria, and Autophagy in Cancer: A Maestro With a Large Orchestra. Front Oncol 2018; 8:73.

63 Fradet Y, Picard V, Bergeron A, LaRue H. Cancer-testis antigen expression in bladder cancer. Prog Urol 2005; 15.URL https://pubmed.ncbi.nlm.nih.gov/16734221/ [accessed on 12 January 2021].

64 Djureinovic D, Hallström BM, Horie M, et al. Profiling cancer testis antigens in non-small-cell lung cancer. JCI Insight 2016; 1:e86837.

65 Grizzi F, Franceschini B, Hamrick C, et al. Usefulness of cancer-testis antigens as biomarkers for the diagnosis and treatment of hepatocellular carcinoma. J Transl Med 2007; 5:3.

66 Tsang M, Gantchev J, Netchiporouk E, et al. A study of meiomitosis and novel pathways of genomic 
instability in cutaneous T-cell lymphomas (CTCL). Oncotarget 2018; 9:37647-61.

2
67 Gantchev J, Martínez Villarreal A, Gunn S, et al. The ectopic expression of meiCT genes promotes meiomitosis and may facilitate carcinogenesis. Cell Cycle 2020; 19:837-54.

68 Sivanand A, Hennessey D, Iyer A, et al. The Neoantigen Landscape of Mycosis Fungoides. Front Immunol 2020; 11. doi:10.3389/fimmu.2020.561234.

69 Brown D, Smeets D, Székely B, et al. Phylogenetic analysis of metastatic progression in breast cancer using somatic mutations and copy number aberrations. Nat Commun 2017; 8:14944.

70 Reiter JG, Makohon-Moore AP, Gerold JM, et al. Reconstructing metastatic seeding patterns of human cancers. Nat Commun 2017; 8:14114.

(1)

5

6

7




\section{Figure legends}

Figure 1. Summary of methods and study design. 32 biopsies of lesional skin were obtained from 23 patients, consisting of 14 patients that contributed single biopsies and 8 patients where multiple samples were taken. The lesions were categorized according to the both the clinical stage (advanced vs. early) as well as the morphological feature (plaque vs lesion): ESP ( $n=12$ early stage plaque biopsies from patients in stage IA-IIA), LSP and TMR ( $\mathrm{n}=10$ late-stage plaques and 15 tumors biopsies from patients in stage $\geq$ IIB, respectively). After RNA-Seq, reads were aligned to the reference sequence after removal of adapter sequences using Bowtie2. Transcript abundance was measured using RSEM. Two statistical methods were used for differential expression analysis and principal coordinate analysis (DESEq2 and edgeR, respectively). Generally applicable gene set enrichment (GAGE) analysis was performed to provide more information about the biological functions and pathways significantly enriched in each disease stage.

Figure 2. Comparative transcriptome analysis of ESP, LSP, and TMR lesions. A. Unsupervised Principal coordinate analysis (PoCA) showing the variation and clustering among ESP, LSP, and TMR lesion types, with ellipses drawn manually. LSP resembles TMR (clusters B1, B2), rather than ESP (cluster A). B. Volcano plot of differential expressed genes (DEGs) between TMR (comparison) and ESP (reference) group, generated using the EnhancedVolcano R package. The vertical y-axis) corresponds to the mean expression value of $\log 10$ (adjusted $\mathrm{P}$ value) after correction for multiple testing, and the horizontal $\mathrm{x}$-axis displays the $\log 2$ fold change value. 1,154 transcripts with an adjusted p-value $<0.05$ and $\mid \log 2$ (fold change) $\mid \geq 1$ were mapped as DEGs (depicted as red dots). Genes that failed to reach statistical significance are present in green, while transcripts below fold-change cutoffs are presented in blue. Genes upregulated or downregulated in TMR compared to ESP are in the upper right and upper left quadrants, respectively. C. Volcano plot for the distribution of gene expression between LSP (comparison) and ESP (reference), mapping 26 DEGs (red dots). D. Volcano plot for the distribution of gene expression between TMR (comparison) and LSP (reference), mapping 29 DEGs (red dots).

Figure 3. Venn diagrams analysis of differentially expressed genes. The yellow, red and green circles represent the number of up or down-regulated DEGs between each pairwise comparison (TMR vs. ESP, TMR vs. LSP, and LSP vs. ESP). The overlapping number represents mutual, concordantly changed DEGs common to each comparison and the non-overlapping number represents genes unique to each comparison. 
1 Figure 4. KEGG Pathway and GO enrichment analysis of early (ESP) and advanced staged MF

2 (LSP or TMR) using all available gene expression data. A. Heatmaps of select enriched KEGG

3 pathways and GO biological process terms (rows) that are significantly over-represented in TMR vs ESP.

4 B. Heatmaps of select enriched KEGG pathways and GO biological process terms (rows) that are

5 significantly over-represented in LSP vs ESP. C. Venn diagram showing GO and KEGG annotations

6 common to both LSP and TMR relative to ESP, notably 'response to interleukin-4', 'respiratory burst',

7 'TOR signaling', 'DNA replication' and 'base excision repair'. KEGG, Kyoto Encyclopedia of Genes and

8 Genomes; GO, gene ontology.

10 Figure 5. Proposed mechanism of stage progression in MF. A. Frequently mutated genes in advanced

11 lesions of MF. The frequencies of non-synonymous mutations (missense or damaging: frameshift

12 mutations, short read insertions and deletions ( $<6 \mathrm{pm})$, stop gain or stop loss) are shown. Only genes

13 showing compensatory overexpression (significantly increased $\log 2 \mathrm{FC}>1$, adjusted $\mathrm{P}<0.05 \mathrm{mRNA}$

14 levels in TMR vs ESP) are shown. B. Possible involvement mutated genes and upregulated signaling

15 pathways during stage progression. The pathways are marked by red ellipses, mutated genes affecting the

16 pathway are plotted in yellow rectangles. $\mathbf{C}$. The proposed mechanism of propagation of mutated cells to

17 skin lesions. Early stage plaques contain cells characterized by a low mutation burden ${ }^{17}$ and a

18 characteristic transcriptional signature as shown in Figure 2. Accumulation of mutations (colored dots in

19 the "nuclei") changes the transcriptional signature which enables higher rate of proliferation and

20 enhanced cell survival which lead to the formation of a tumor (high-grade malignant cells marked in red).

21 Those cells can enter the circulation, e.g. due to loss of expression of CX3CR1 and can colonize the

22 already existing plaques (cancer self-seeding) or new areas of the skin in a quasi-metastatic process as

23 proposed by us previously ${ }^{14}$. 


\section{4 patients}

Single samples

W 10 ESP 2 LSP

2 TMR

8 patients

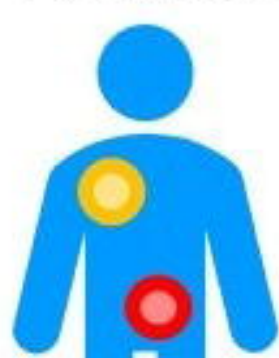

Multiple samples

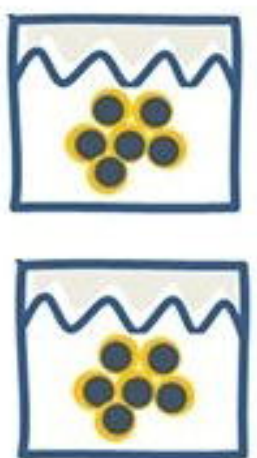

2 ESP

8 LSP 13 TMR

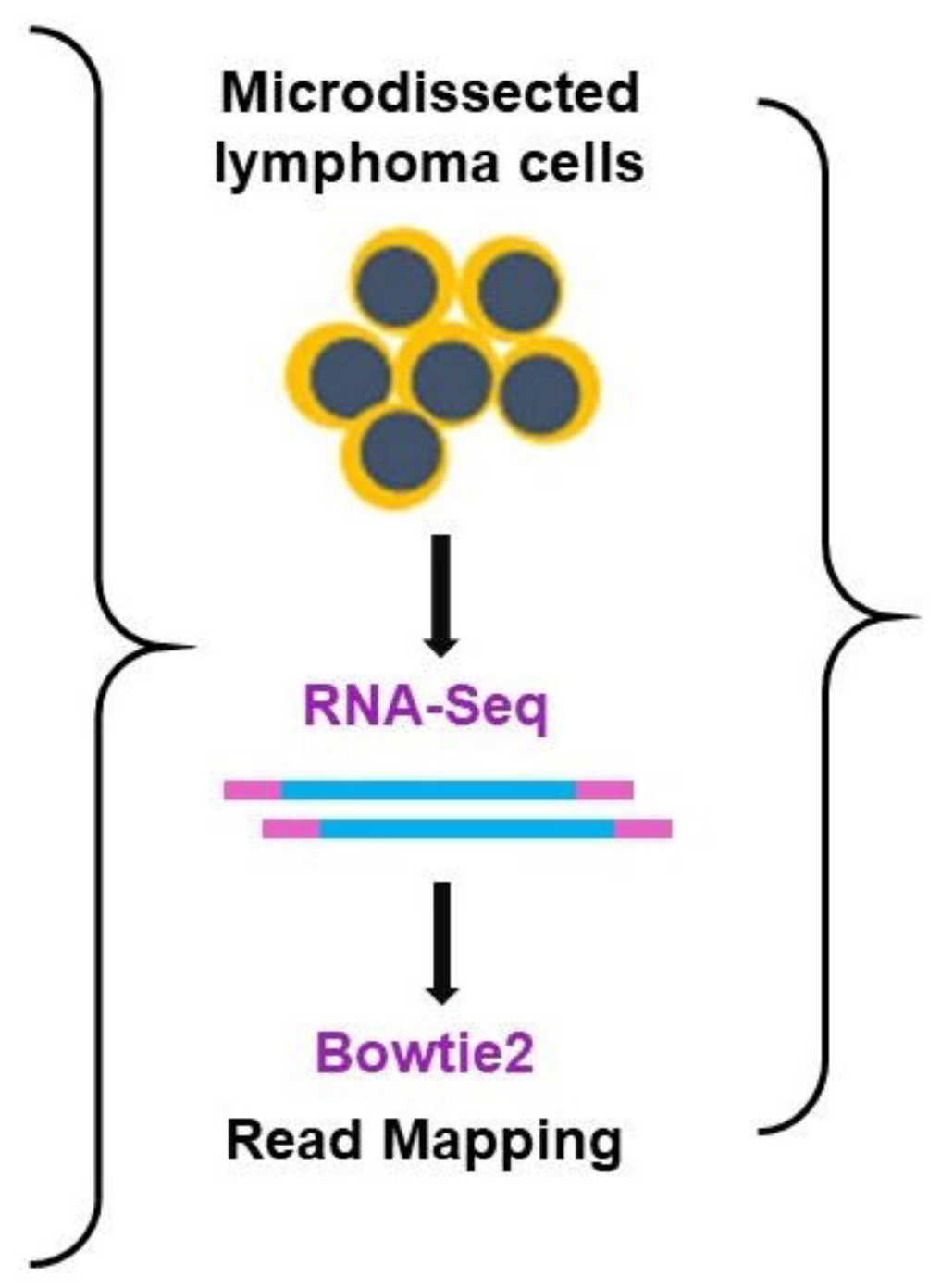

Read Mapping
RSEM

Abundance Estimation

I

DESeq2

Differential

Expression

Analysis

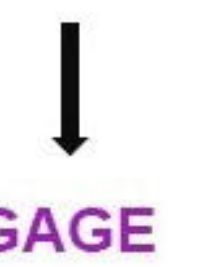

KEGG Pathway \& GO Analysis

$$
\text { edge R }
$$

Principal Coordinate Analysis

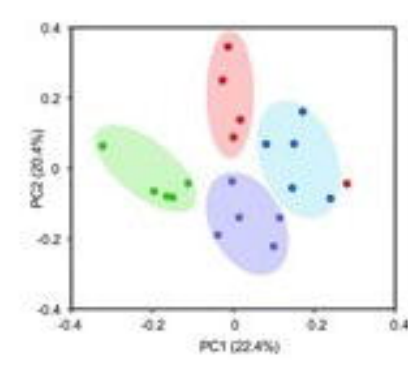




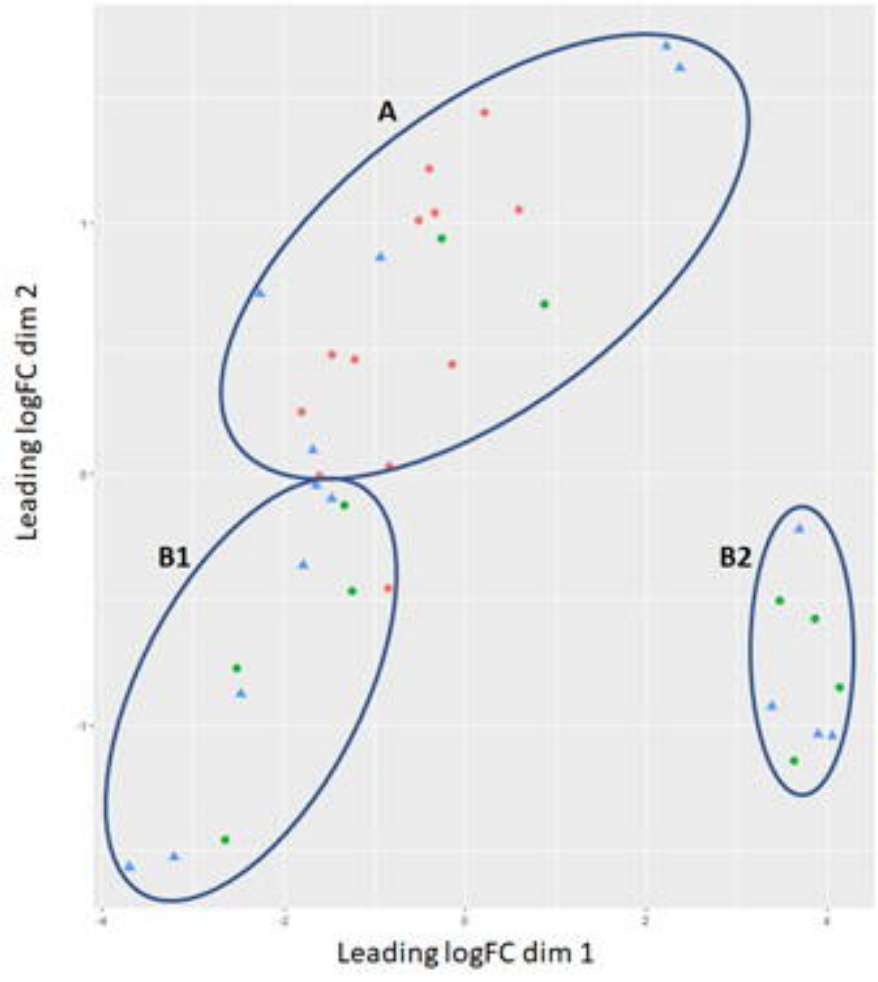

LSP versus ESP

- NS $-\log _{2} \mathrm{FC}$ - $\mathrm{p}$-value and $\log _{2} \mathrm{FC}$

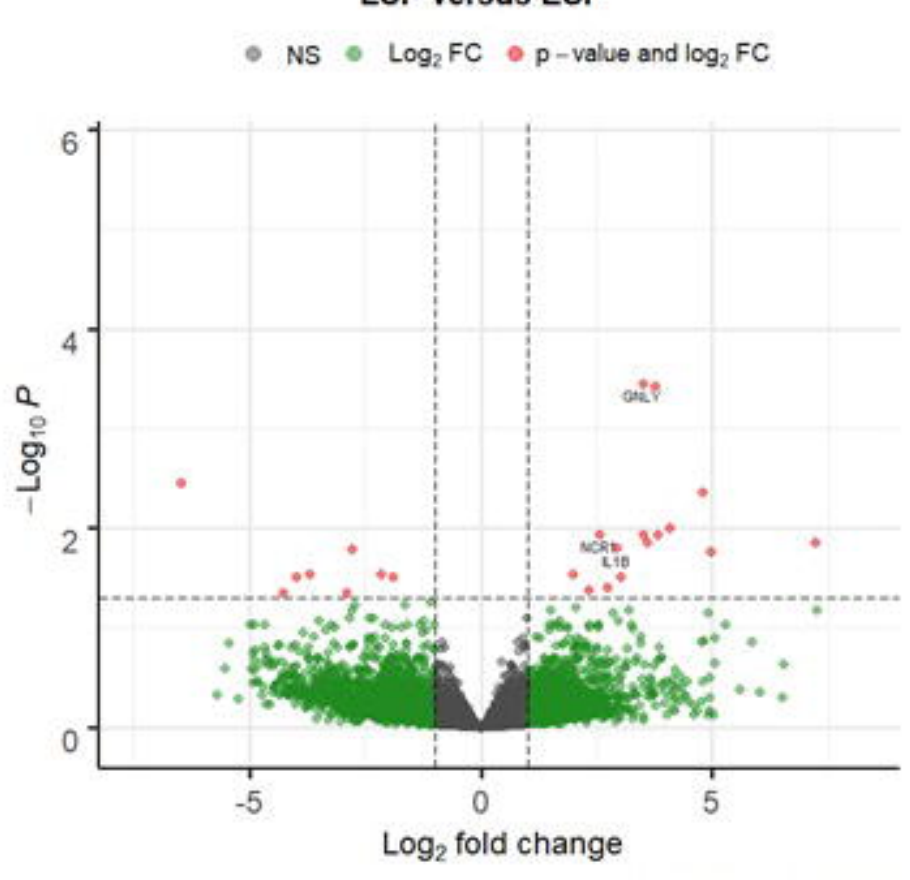

Total $=60561$ variables

Lesion

- Plaque

- Tumor

Disease Stage

- Esp

- เงe

- tur

\section{TMR versus ESP}

- NS $-\log _{2} \mathrm{FC} \bullet \mathrm{p}$-value $\mathrm{p}$-value and $\log _{2} \mathrm{FC}$

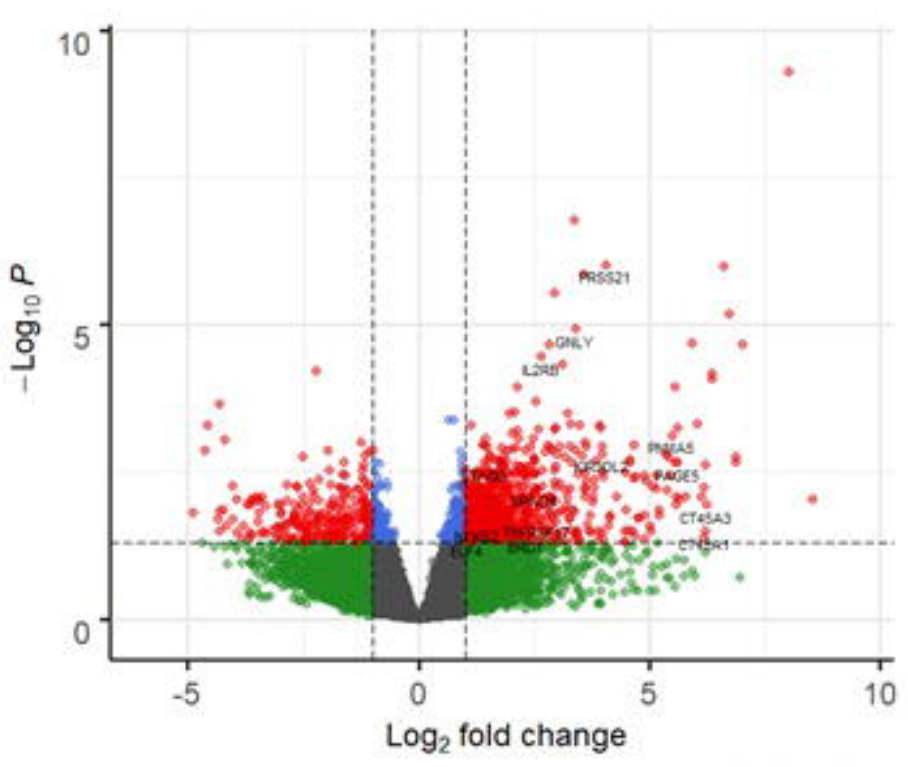

Total $=60561$ variables

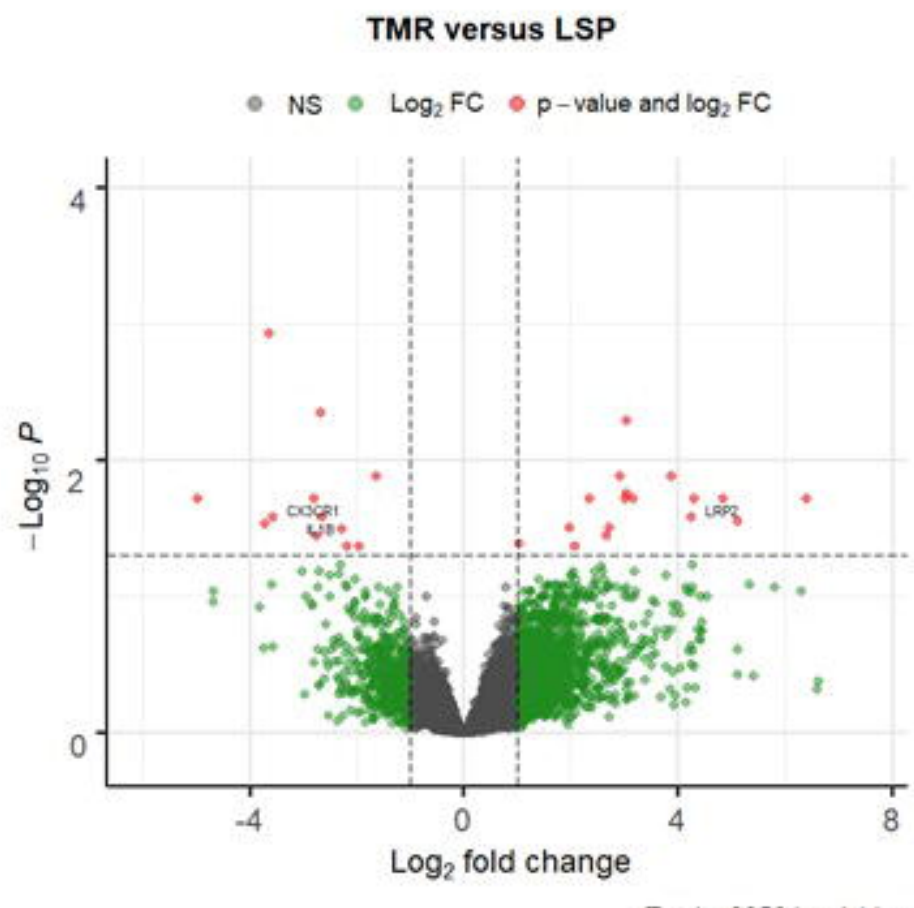




\section{UP}

TMR vs. ESP

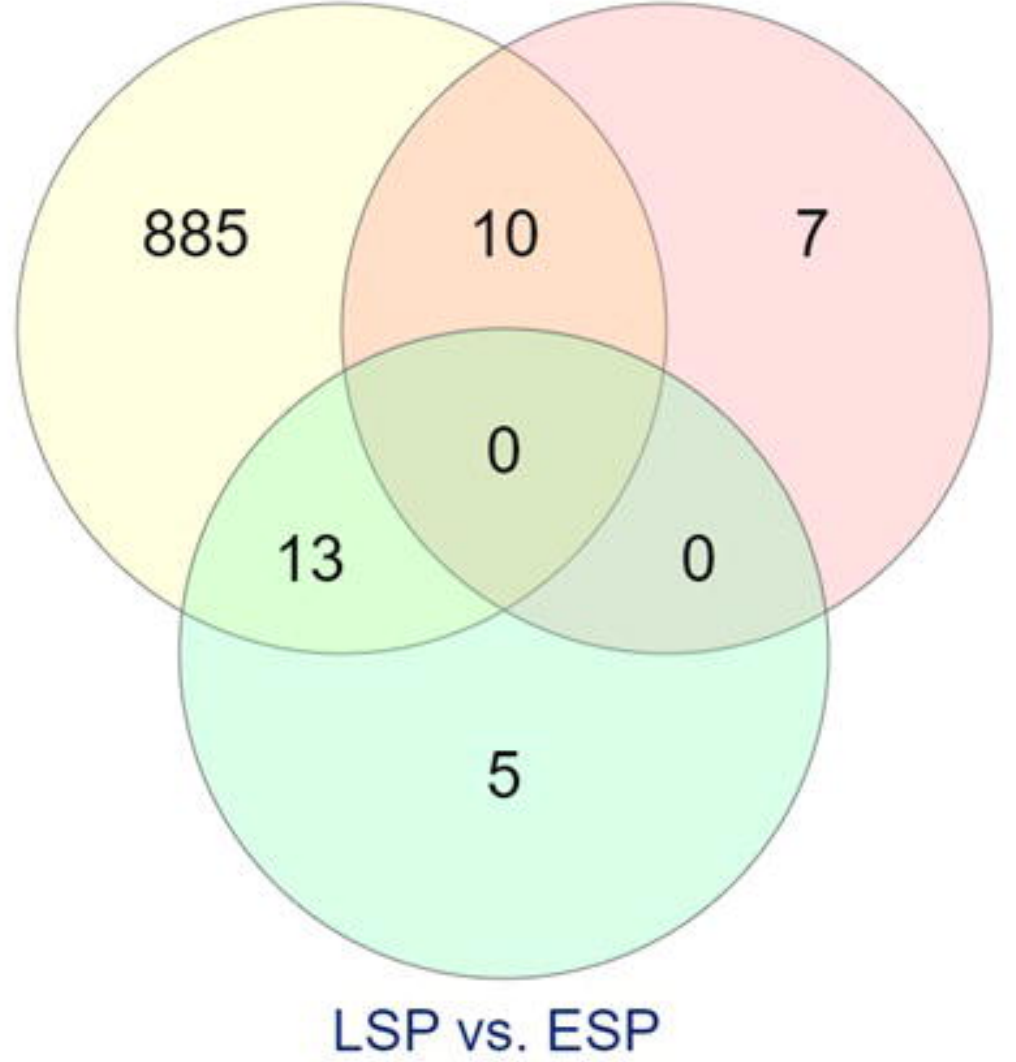

DOWN

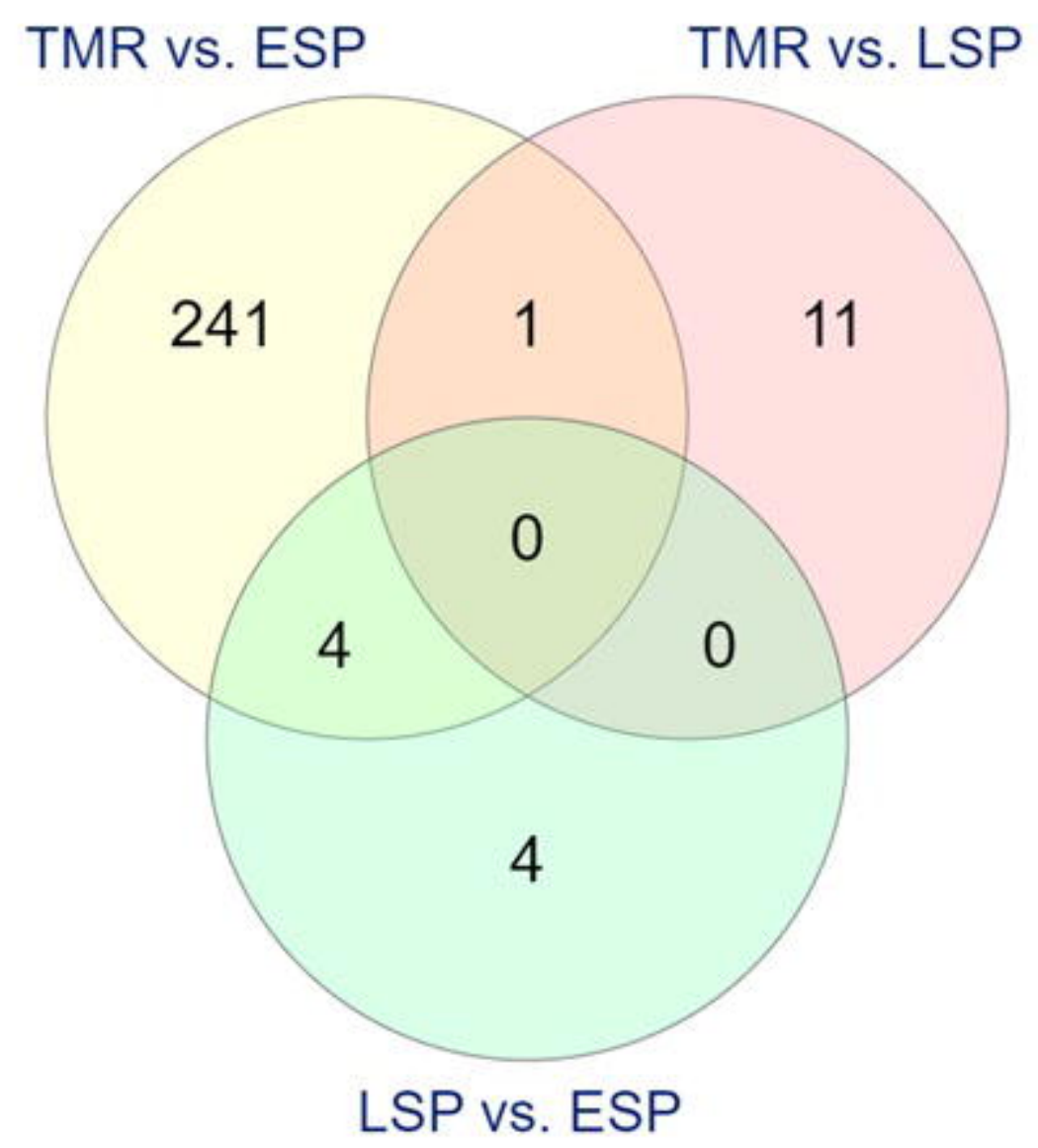




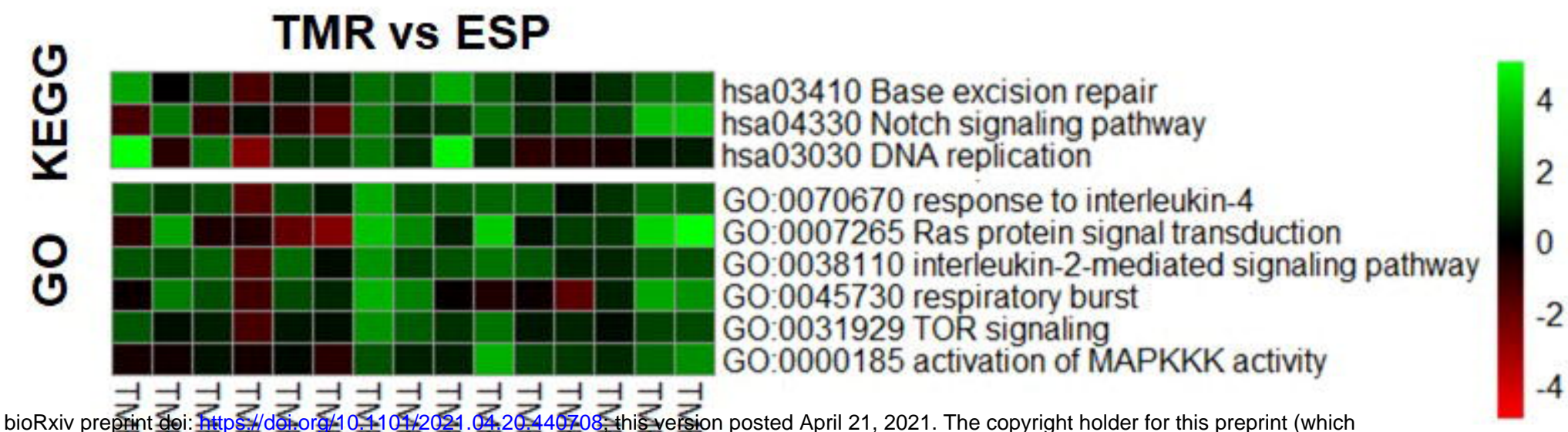

was notcertilied by peer deview) is thelauthor/funder, wbo bas dranted bioRxiv a license to display the preprint in perpetuity. It is made

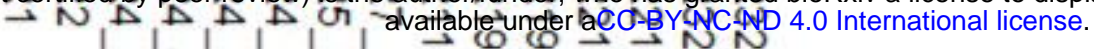

B

LSP vS ESP

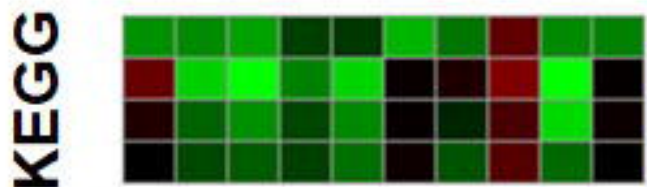

hsa04660 T cell receptor signaling pathway

hsa04110 Cell cycle

hsa03030 DNA replication

hsa03410 Base excision repair

GO:0045730 respiratory burst

GO:0071353 cellular response to interleukin-4

GO:0036297 interstrand cross-link repair

GO:0006289 nucleotide-excision repair

$\underset{0}{0}$

GO:0006298 mismatch repair

GO:0007257 activation of JUN kinase activity

GO:0038066 p38MAPK cascade

GO:0038202 TORC1 signaling

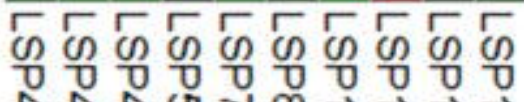

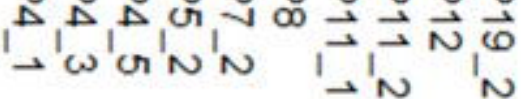

\section{LSP vs. ESP}

TMR vs. ESP

p38MAPK cascade $\neq$

T-cell receptor signaling

Pathway $\S$

Nucleotide-excision

Base excision repair §

Repair ¥

Activation of JUN

kinase activity $¥$

Interstrand cross-link repair ¥

Response to IL-4 ₹

Respiratory burst ₹

DNA replication $\S$

TOR signaling $\ddagger$
IL-2 mediated signaling ₹

Notch signaling pathway $\S$

Ras protein signal transduction ¥

Activation of MAPKKK activity $¥$

Mismatch repair ¥

Cell cycle $\S$

$\S$ KEGG pathway ‡ GO term 
A

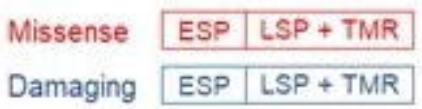

Frequency of mutations ( $\%$ )

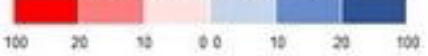

\begin{tabular}{|c|c|}
\multicolumn{1}{|c|}{ MED12 } \\
\hline $8 \%$ & $27 \%$ \\
\hline $0 \%$ & $9 \%$ \\
\hline
\end{tabular}

\begin{tabular}{|c|c|}
\hline \multicolumn{2}{|c|}{ CIC } \\
\hline $17 \%$ & $5 \%$ \\
\hline $25 \%$ & $41 \%$ \\
\hline
\end{tabular}

\begin{tabular}{|c|c|}
\hline \multirow{2}{*}{\multicolumn{2}{|c|}{$\begin{array}{l}\text { CARD11 } \\
8 \% 9 \%\end{array}$}} \\
\hline & \\
\hline & \\
\hline
\end{tabular}

\begin{tabular}{|l|l|}
\multicolumn{2}{|c|}{ JAK3 } \\
\hline $0 \%$ & $5 \%$ \\
\hline $8 \%$ & $14 \%$ \\
\hline
\end{tabular}

C

\section{Stage I}

Early Stage Plaque

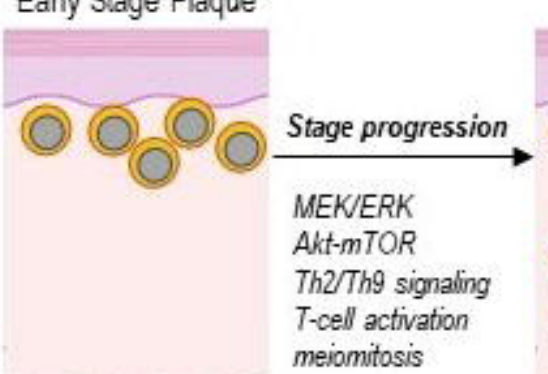

B

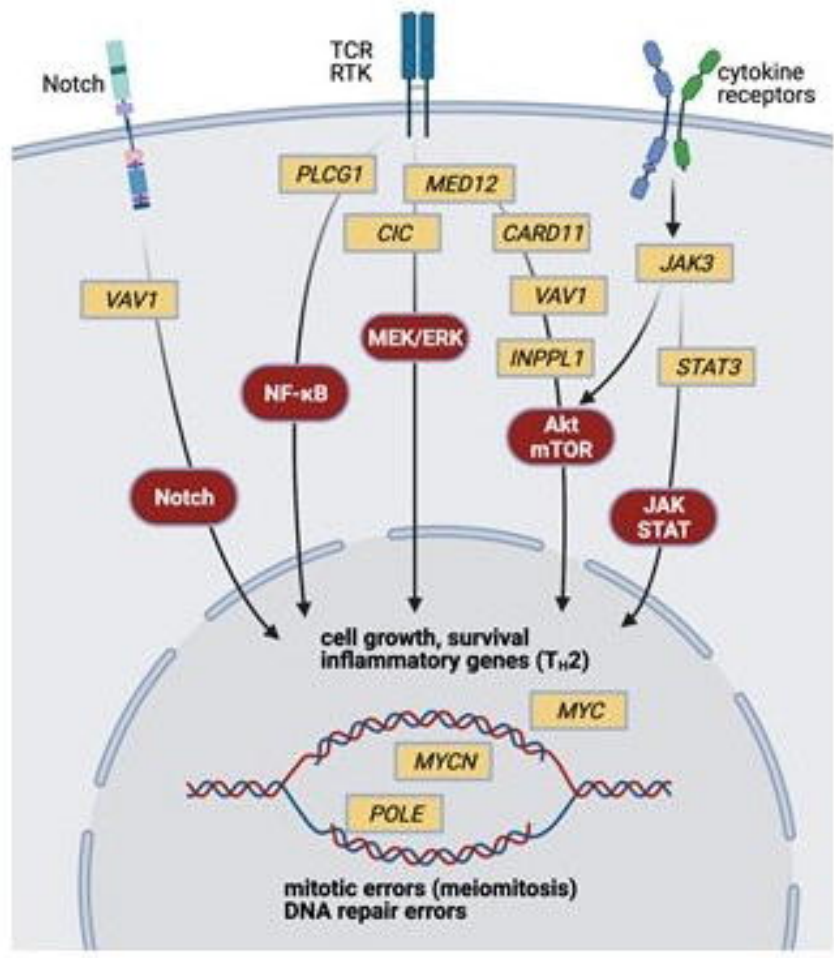

\section{Stage II}

Tumor

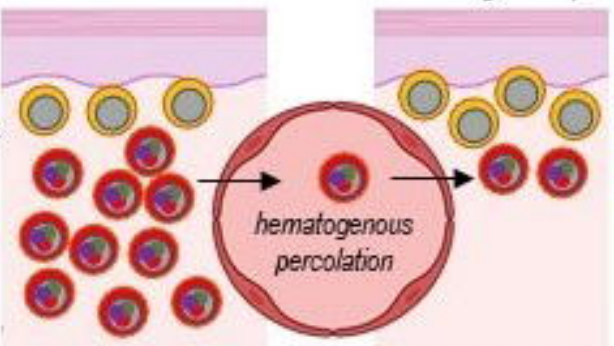

